\title{
Cardiac-specific overexpression of E40K active Akt prevents pressure overload-induced heart failure in mice by increasing angiogenesis and reducing apoptosis
}

\author{
Cell Death and Differentiation (2007) 14, 1060-1062. doi:10.1038/sj.cdd.4402095; published online 19 January 2007
}

Dear Editor,

Physiological myocardial hypertrophy is the result of adaptation of the heart to increased work demands on the cardiovascular system. This response is mediated by pathways which trigger increased protein synthesis and the consequent growth in cardiomyocyte (CMC) size. Multiple signaling pathways contribute to the physiological hypertrophy phenotype, of which one well studied is the IGF1-PI3K-Akt pathway. ${ }^{1}$ This pathway is also involved in the mechanism of action of pro-angiogenic factors like vascular endothelial growth factor (VEGF), and leads to capillary formation and migration. ${ }^{1,2}$

We previously demonstrated that cardiac-specific overexpression of a constitutively active mutant of Akt (E40K-Akt) induces not only a physiological type of hypertrophy but also improvements in cardiac function by increasing L-type $\mathrm{Ca}^{2+}$ current and sarcoplasmic reticulum pump (SERCA) activity. ${ }^{3}$ Accordingly, others found that with knockout of Akt- $1^{4}$ or overexpression of a dominant-negative $\mathrm{PI} 3 \mathrm{~K} \alpha$ mutant, ${ }^{5}$ the physiological myocardial adaptations and hemodynamic improvements consequent to physical stress were prevented.

Induction of pressure overload through transverse aortic constriction (TAC) is an established technique that permits the study of the effects of gene overexpression or knockout in mice undergoing heart failure. Models of overexpression of Akt in CMCs present with significant phenotypic variability (reviewed by Catalucci et al. ${ }^{3}$ ). Constitutive or long-term overexpression of a myristilated (myr) form of Akt in CMCs were associated with negative effects on inotropism, either inducing heart failure or worsening cardiac function. ${ }^{2}$ The negative effects of myr-Akt on cardiac function were reverted by expression of a constitutively active form of $\mathrm{PI} 3 \mathrm{~K} \alpha{ }^{6}$ In contrast, cardiac-specific overexpression of myr-Akt improved cardiac function in the short-term: ${ }^{2}$ here, Akt overexpression was accompanied by increased angiogenesis and VEGF protein expression. ${ }^{2}$ Another interesting model of Akt overexpression is represented by cardiac-specific overexpression of a nuclear form of Akt, characterized by increased resistance to apoptosis and improved cardiac function. ${ }^{7}$

We therefore assessed the effects of cardiac-specific overexpression of the E40K-Akt mutant on cardiac function in the TAC model of heart failure. We found that Akt ameliorates cardiac function by decreasing apoptosis and fibrosis and improving angiogenesis.
Transgenic mouse had significantly better cardiac function than WT after TAC during the course of our study (Figure 1a and Supplementary Tables 1-2, Supplementary Information). Phosphorylation of Akt at S473 in WT decreased significantly (three-/four-fold) only after 8 weeks of TAC. Levels in Tg mice were always greater (five-fold) than WT and never changed significantly (Figure 1b). Fetal gene expression was induced in both WT and in Tg mice after 1 week of TAC, but remained elevated at 8 weeks only in Tg mice (Figure 1c).

We quantified capillaries in histological sections of hearts stained with FITC-conjugated lectin $^{8}$ (Figure 1d, top). Under basal conditions, vascularization of $\mathrm{Tg}$ mouse myocardium was $1.80 \pm 0.15$ capillaries/CMC as compared to $1.15 \pm 0.18$ capillaries/CMC in WT controls (Figure 1d, bottom). One week after TAC, capillary density increased significantly in the myocardium of WT mice ( $1.52 \pm 0.06$ capillaries/CMC), thus accompanying the adaptive hypertrophic response. Longterm exposure ( 8 weeks) to pressure overload did not induce any further increase of capillaries/CMC. In Tg mice, however, the baseline capillary/CMC ratio was greater than that found in WT mice, and did not change significantly, neither during the first week of banding nor after 8 weeks.

As VEGF correlates with capillary density, ${ }^{2,9}$ we studied VEGF levels. To this end, qRT-PCR was first used to investigate VEGF gene expression (Figure 1e, top). Results show that in WT mice, VEGF mRNA was increased after 1 week of TAC and then returned to baseline at 8 weeks. No significant variation could be detected in $\mathrm{Tg}$ mice. Western blotting was then performed to investigate VEGF at the protein level (Figure 1e, bottom). In WT mice, VEGF increased significantly (nine-fold) 1 week after TAC, then dropped significantly (1.5-fold) from this level at 8 weeks. In contrast, levels were higher in Tg mice (seven-fold) than in WT controls, and did not change after TAC.

Apoptosis is considered an important event during the early phases of heart failure. ${ }^{10}$ Consequently, we compared apoptosis in the myocardium of WT and Tg mice after TAC with TUNEL (Figure 1f, left). Baseline levels of apoptotic cells are normally negligible in healthy myocardium; in fact, we found rare TUNEL-positive nuclei both in WT and in Tg mice (Figure 1f, right). In WT mice, apoptotic events in the myocardium were increased after 1 week of TAC and greatly 
a

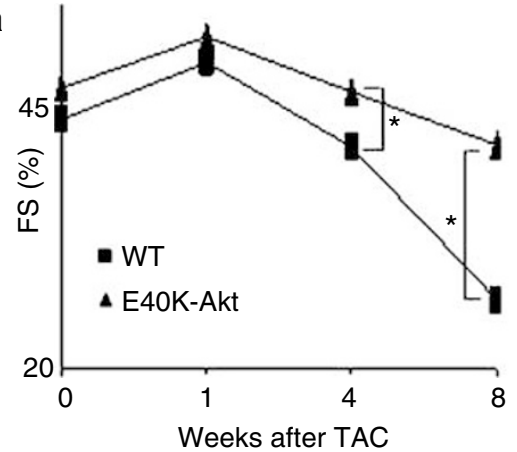

b

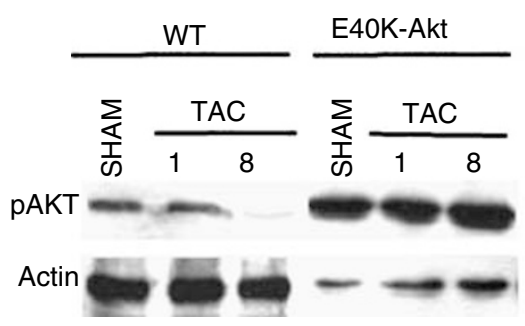

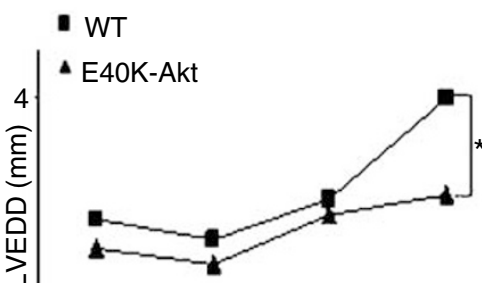
1.5

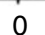

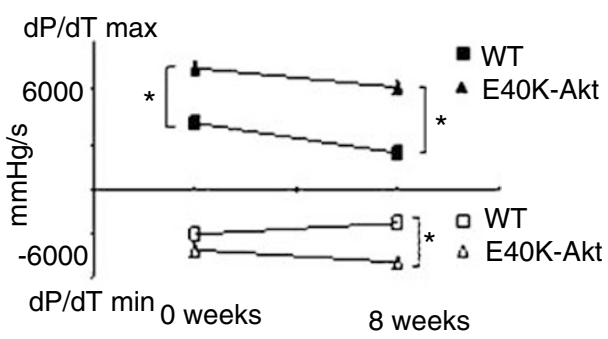

c

ANF

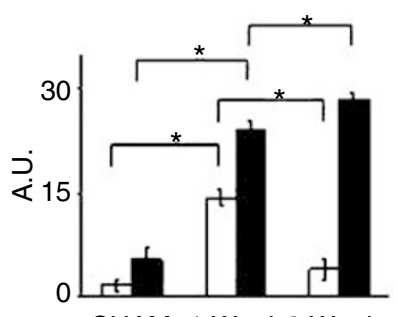

SHAM 1 Week 8 Weeks
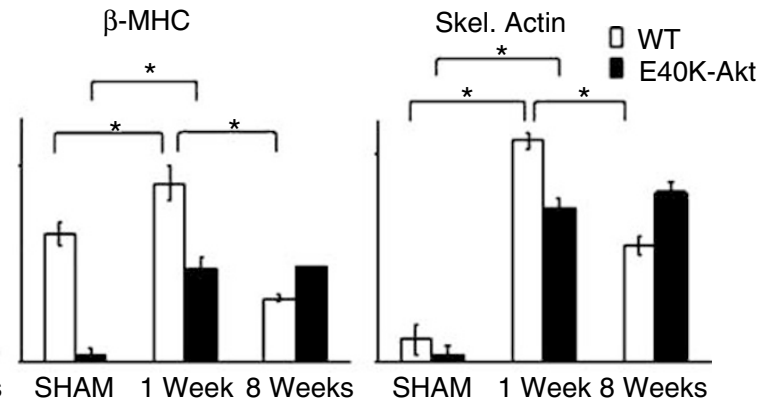

d
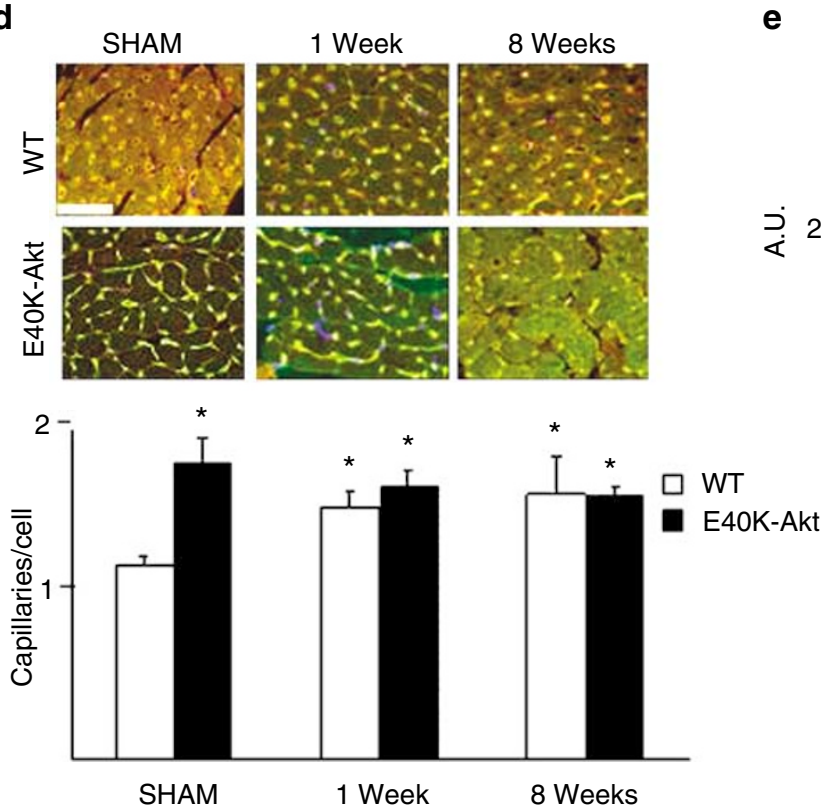

e

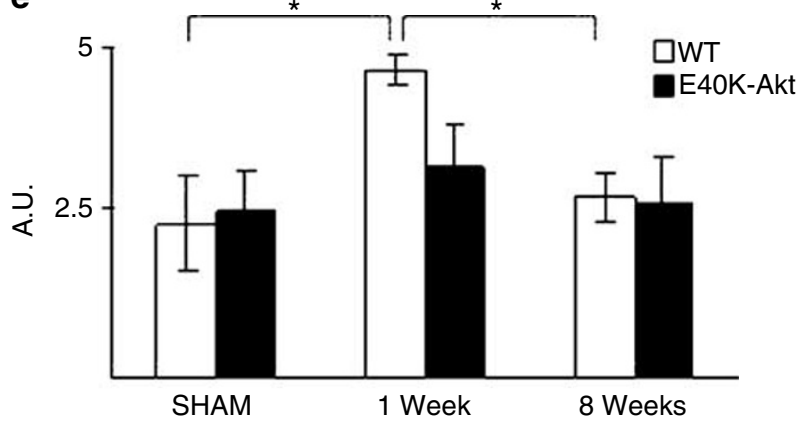

f

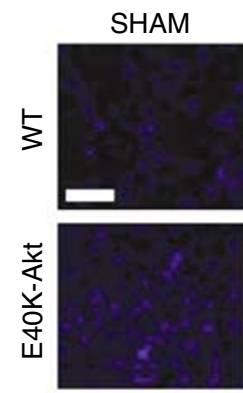

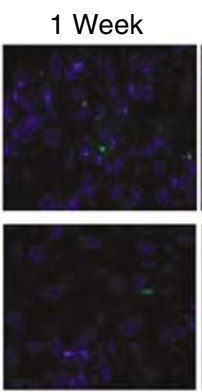

8 Weeks

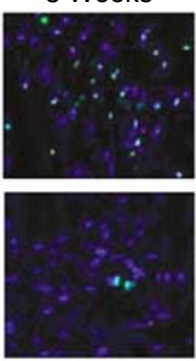

Figure 1

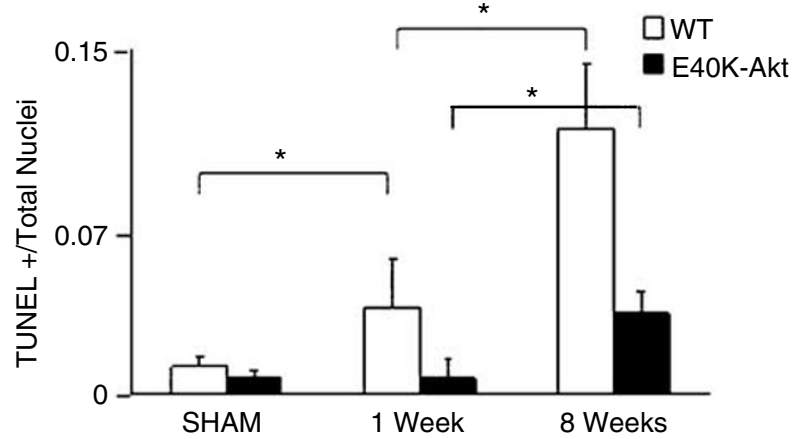


Figure 1 Heart function and the effects of Akt overexpression before and after TAC: (a) (left) Time course of LV fractional shortening (FS) in WT and Tg versus duration of TAC; ${ }^{*} P<0.05$. (middle) Time course of LV end diastolic diameter (LVEDD) in WT and Tg versus duration of TAC; ${ }^{*} P<0.05$. (right) LV dP/dT in WT and Tg before and after 8 weeks of TAC; ${ }^{*} P<0.05$. (b) Representative Western blot showing phosphorylation of Akt at serine 473 in heart extracts from sham-operated and TACoperated WT and Tg mice (1 and 8 weeks after TAC). (c) qRT-PCR analyses of fetal gene expression versus duration of TAC (mean \pm S.D.) (ANF, atrial natriuretic factor; $\beta$-MHC, $\beta$-myosin heavy chain; Skel. Actin, skeletal actin); ${ }^{*} P<0.05$. (d) (top) Representative images of CMCs stained against isolectin B4 lectin and counterstained with wheat germ agglutinin; scale bar $=50 \mu \mathrm{m}$. (bottom) Bar graph of ratio of capillaries to nuclei (mean \pm S.D.); ${ }^{*} P<0.05$ versus WT sham. (e) (top) qRT-PCR analysis of VEGF mRNA in sham and TAC-operated WT and Tg mice (1 and 8 weeks after TAC) (mean \pm S.D.); ${ }^{-} P<0.05$. (bottom) Representative Western blot of time course of VEGF protein in WT and Tg mice after TAC. Data were obtained from four animals each strain. (f) (left) Representative images of histological sections assayed for TUNEL-positive nuclei from WT and Tg mice; scale bar $=50 \mu \mathrm{m}$. (right) Bar graph of ratio of TUNEL-positive nuclei to total number of nuclei (mean \pm S.D.); ${ }^{*} P<0.05$

increased after 8 weeks: the TUNEL-positive nuclei were seen in clusters. In contrast, Tg mice seemed protected from apoptosis, as the number of TUNEL-positive nuclei increased only slightly after 1 week of TAC and remained at low levels thereafter. At 8 weeks, Tg mice had three-fold less apoptotic cells than their respective WT counterparts.

Fibrosis is another feature of the response to TAC, and becomes evident in WT after 8 weeks. It was never encountered in $\mathrm{Tg}$ mice (Figure 1 Supplementary Information).

Thus, the pro-hypertrophic and antiapoptotic effects of Akt are not mutually exclusive. In fact, while inducing hypertrophy, overexpression of the E40K-Akt mutant is capable of preventing apoptosis of cardiac myocytes during pressure overload. This effect may well depend on both nuclear and cytoplasmic Akt localization and phosphorylation of relative substrates. In fact, cytoplasmic substrates of Akt involved in improving cardiac function include, among others, FOXO3 transcription factor ${ }^{11}$ which, upon phosphorylation, is prevented from entering the nucleus and activating transcription of genes involved in protein catabolism or mitochondrial proteins involved in survival. ${ }^{12}$ The pro-angiogenic effect of Akt overexpression can account at least partly for its antiapoptotic effect during pressure overload hypertrophy: beside its angiogenic effect, VEGF is a potent antiapoptotic agent for CMCs in in vivo models of cardiomyopathies. In fact, replenishment of VEGF levels using naked DNA gene therapy via direct intramyocardial injection of plasmid DNA encoding VEGF (phVEGF165) induced increased capillary density and decreased CMC apoptosis. ${ }^{13}$

Absence of the upstream Akt activator PDK-1 in cardiac myocytes dramatically decreases the ability of CMCs to cope with hypoxia. ${ }^{14}$ More recently, the absence of Akt1 in myocardial tissue was associated with worsening of cardiac function during pressure overload stress. ${ }^{4}$ These two reports strongly suggest that Akt is necessary for preventing cellular damage also during increased workload.

Results of our as well as of other groups suggest that both nuclear and cytoplasmic localization are critical for Akt's antiapoptotic effects. The anti-hypertrophic effects of nuclear Akt were suggested to be dependent on gene expression of ANP, ${ }^{15}$ which, upon an autocrine-paracrine effect, stimulates cGMP production and downstream signal transduction activation, leading to hypertrophy prevention. Such an effect seems also to account for the anti-hypertrophic effect of 17- $\beta$-estradiol, which also activates Akt, leading to cGMP production and antagonizing hypertrophy, ${ }^{16,17}$ although activation of $\mathrm{PI}-3 \mathrm{~K} \alpha$ through direct interaction with estrogen receptor is another possible activation mechanism. ${ }^{18,19}$ Although the role of Akt in establishing physiological hypertrophy is well known, whether it has an anti-apoptotic effect also during this condition needs further investigation.

Acknowledgements. GC is supported by grants from the $\mathrm{NIH}$ (RO HL078797-01A1), the European Community (EU FP6 grant LSHM-CT-2005018833, EUGeneHeart), Italian Ministry of Health, Italian Ministry of Research and University. DC is supported by a Marie Curie International Fellowship within the 6th European Framework Programme.

\section{Ceci, ${ }^{1,6}, P$ Gallo $^{2,3,6}, M$ Santonastasi ${ }^{1,3,6}, S$ Grimaldi $^{1,3}$, MVG Latronico ${ }^{1}$, A Pitisci $^{1,3}$, E Missol-Kolka $^{4}$, MC Scimia ${ }^{1,5}, D$ Catalucci, $^{1,5}, D$ Hilfiker-Kleiner ${ }^{4}$ and $\mathrm{G}$ Condorelli*,1,5}

1 I.R.C.C.S. Multimedica, Milan, Italy

2 University Campus Biomedico, Rome, Italy

${ }^{3}$ San Raffaele Biomedical Science Park Foundation, Rome, Italy

4 Department of Cardiology and Angiology, Hannover Medical School, Hannover, Germany

${ }^{5}$ Department of Medicine, Division of Cardiology, University of California San Diego, La Jolla, USA

6 These authors contributed equally to this work

* Corresponding author: G Condorelli, Division of Cardiology, Department of Medicine, University of California San Diego, La Jolla, CA, 92093-0734, USA. Tel: + 18585343347; Fax: + 18585341626; E-mail: gcondorelli@ucsd.edu

1. Walsh K (2006) Circulation 113: 2032-2034

2. Shiojima I et al. (2005) J Clin Invest 115: 2108-2118.

3. Catalucci D et al. (2006) Circ Res 99: 339-341.

4. DeBosch B et al. (2006) Circulation 113: 2097-2104.

5. McMullen JR et al. (2003) Proc Natl Acad Sci USA 100: 12355-12360.

6. Nagoshi T et al. (2005) J Clin Invest 115: 2128-2138.

7. Rota M et al. (2005) Circ Res 97: 1332-1341.

8. Hilfiker-Kleiner D et al. (2005) Circulation 112: 1470-1477.

9. Shiojima I et al. (2002) Circ Res 90: 1243-1250.

10. Foo RS et al. (2005) J Clin Invest 115: 565-571.

11. Skurk $C$ et al. (2005) J Biol Chem 280: 20814-20823.

12. Uchiyama T et al. (2005) Circulation 111: 2073-2085.

13. Yoon YS et al. (2005) Circ Res 111: 2073-2085.

14. Mora A et al. (2003) EMBO J 22: 4666-4676.

15. Tsujita $Y$ et al. (2006) Proc Natl Acad Sci USA 103: 1946-1951.

16. van Eickels M et al. (2001) Circulation 104: 1419-1423.

17. Babiker FA et al. (2004) Circulation 109: 269-276.

18. Patten RD et al. (2004) Circ Res 95: 692-699.

19. Simoncini T et al. (2000) Nature 407: 538-541.

Supplementary Information accompanies the paper on Cell Death and Differentiation website (http://www.nature.com/cdd) 